\title{
Dolor como factor predictor de depresión en el paciente oncológico: estudio de casos y controles. Estudio D-PRESS
}

\author{
J. Carulla ${ }^{1}$, C. Jara ${ }^{2}$ J. Sanz ${ }^{3}$, C. Martínez ${ }^{4}$, F. Ledesma ${ }^{4}$ y E. Zubillaga ${ }^{4}$. En nombre del grupo \\ de estudio D-PRESS*
}

${ }^{1}$ Hospital Vall D’Hebron, Barcelona. ${ }^{2}$ Hospital Universitario Fundación de Alcorcon.

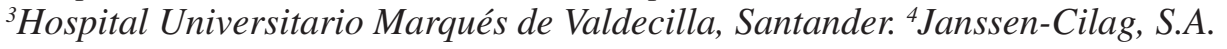

Carulla J, Jara C, Sanz J, Martínez C, Ledesma F, Zubillaga E. Dolor como factor predictor de depresión en el paciente oncológico: estudio de casos y controles. Estudio D-PRESS. Rev Soc Esp Dolor 2013; 20(3): 113-121.

\begin{abstract}
Objectives: To evaluate the relationship between cancer pain and depression, to determine the prevalence of depression in both groups the cases and controls and also to evaluate which factors from the medical records can be potentially associated with depression.

Methods: Observational, retrospective and multi-centre study. Patients were $\geq 18$ years old, had medical data $\geq 3$ months, experienced pain after cancer diagnosis (VAS $\geq 3$ ) and completed the Beck Depression Inventory (BDI). Patients were classified as cases and controls based on the BDI score $\left({ }^{3} 8\right.$ and $£ 7$, respectively).
\end{abstract}

Results: A total of 248 patients were included (mean age $59.0 \pm 12.4$ years). Mean BDI for the case group was $13.1 \pm$ 4.9 , and for the control group, $3.8 \pm 2.1$. Patients in the control group, had a longer survival time (20.0 months) in comparison with patients in the case group (12 months; $p=0.0032$ ).

For the overall population, BDI scoring was correlated with the intensity of pain during the last 24 hours $(0.215 ; \mathrm{p}=$ $0.0007)$ and the hours of pain during the last 24 hours $(0.170$; $\mathrm{p}=0.0073)$.

A more advanced disease stage was also among the factors that increased the risk of depression $(p=0.0113)$.

The multivariate analysis revealed the predictive nature of digestive cancers $(p=0.0454)$ and T stage (T2 vs T4; $p=0.0039$, and T3 vs T4; $p=0.0012$ ) as risk factors for depression.

Conclusions: According with previous studies, there is a clear relationship between pain intensity and patient's psychological status. The predictive potential of medical factors for depression (tumor stage and type of cancer) opens the possibility to target patients at risk.

Key words: Cancer. Depression. Pain.
"El grupo del estudio D-PRESS: A. Sánchez (Hospital Provincial de Castellón), M. Jorge (Hospital Xeral Cíes, Vigo), B. Cantos (Hospital Puerta de Hierro, Madrid), J. Martínez (Hospital Universitario Miguel Servet, Zaragoza), A. Paredes (Hospital de Donostia Osakidetza, Guipúzcoa), J. A. Virizuela (Hospital Universitario Virgen de la Macarena, Sevilla), J. R. Delgado y C. Sánchez (Hospital Universitario Virgen de las Nieves, Granada), E. del Barco (Hospital Universitario de Salamanca), B. Cirauqui y M. Margelí (Hospital Germans Trias I Pujol, Barcelona), J. García (Complejo Hospitalario de

Fuente de financiación: Este trabajo fue financiado por Janssen-Cilag S.A. Conflictos de interés: CM, FL y EZ trabajan para Janssen-Cilag, S.A.

Recibido: 10-06-11.

Aceptado: 26-01-12.
Orense), A. Rizo (Hospital Universitario San Juan de Alicante), C. Carrasquer y J. Pérez (Hospital de La Santa Creu i San Pau, Barcelona), E. Jiménez y J. C. Avanza (Hospital de Jerez, Cádiz), A. L. Yuste (Hospital Universitario de Alicante), J. A. García (Hospital Clínico Universitario San Carlos, Madrid), B. Mellado (Hospital Clinic de Barcelona), S. Casado (Hospital Can Misses, Ibiza), I. C. Barneto (Hospital Universitarios Reina Sofía, Córdoba), S. Escrivá (Hospital de Mollet, Barcelona), J. J. Lambea (Hospital Clínico Universitario de Zaragoza), C. Gómez (Hospital Universitario 12 de Octubre, Madrid), E. Esteban (Hospital Universitario Central de Asturias), J. N. Batista (Hospital Universitario de Canarias), S. Antolín (Hospital Juan Canalejo, La Coruña), S. Enrech (Hospital de Getafe, Madrid), J. M. García (Hospital General de Albacete), A. Redondo (Hospital Universitario La Paz, Madrid), R. Trujillo (Clínica Croasa, Málaga), A. Salud (Hospital Arnau de Villanova, Lérida), M. a A. Vaz (Hospital Ramón y Cajal, Madrid), P. Díaz (Hospital Mateu Orfila, Menorca), M. López-Brea (Hospital Universitario Marqués de Valdecilla, Santander), E. Villar (Hospital Carlos Haya, Málaga). 


\section{RESUMEN}

Objetivos: Valorar la correlación entre dolor oncológico y depresión, determinar la prevalencia de depresión en los grupos de casos y controles, y conocer qué factores de la historia clínica del paciente podrían estar potencialmente asociados a la depresión.

Métodos: Estudio observacional, retrospectivo, y multicéntrico. Se recogió información de la historia clínica de pacientes, de al menos 3 meses anteriores al inicio del estudio, de edad $\geq 18$ años, que padecían dolor tras el diagnóstico oncológico $(E V A \geq 3)$ y que cumplimentaron el inventario de depresión de Beck (IDB). Los pacientes se clasificaron como casos y controles en base a la puntuación IDB ( $\geq 8 y \leq 7$, respectivamente).

Resultados: Se incluyó un total de 248 pacientes con una media de edad de 59,0 $\pm 12,4$ años. La media del IDB para el grupo de casos fue $13,1 \pm 4,9$, y para el grupo control 3,8 $\pm 2,1$. El tiempo medio de supervivencia de los pacientes fue mayor en el grupo control (20,0 meses) en comparación con el grupo caso (12 meses; $p=0,0032$ ). En líneas generales, la puntuación del IDB se correlacionó significativamente con la intensidad del dolor en las últimas 24 horas $(0,215 ; p=0,0007)$ y con el número de horas de dolor durante las últimas 24 horas $(0,170 ; p=0,0073)$.

Presentar un estadio tumoral más avanzado fue uno de los factores que aumentó el riesgo de padecer depresión ( $\mathrm{p}=$ 0,0113).

El análisis multivariante demostró el carácter predictivo del cáncer digestivo $(\mathrm{p}=0,0454)$ y del estadio tumoral T (T2 vs T4; $\mathrm{p}=0,0039$, y T3 vs T4; $\mathrm{p}=0,0012)$ como factores de riesgo de padecer depresión.

Conclusiones: De acuerdo a otros estudios previos, existe una clara correlación entre la intensidad de dolor y el estado psicológico del paciente. El potencial predictivo de ciertos factores clínicos (estadío tumoral y ciertos tipos tumorales) posibilita la identificación de pacientes oncológicos en riesgo de padecer depresión.

Palabras clave: Cáncer. Depresión. Dolor.

\section{INTRODUCCIÓN}

El dolor oncológico está presente en el $90 \%$ de los pacientes con cáncer en estadios avanzados (1). La etiología del dolor depende tanto del tumor en sí mismo (debido a la compresión, oclusión vascular, afectación nerviosa, etc.) como del tratamiento asociado (quimioterapia, radioterapia, etc.). El control del dolor representa un componente muy importante del cuidado global del paciente oncológico. Las decisiones acerca de su tratamiento y la falta de un control apropiado pueden desencadenar otros síntomas asociados al cáncer, como por ejemplo, náuseas, fatiga, disnea, o deterioro cognitivo. Además, existen muchas evidencias de que un tratamiento inapropiado para el dolor disminuye la calidad de vida del paciente así como su esperanza de vida (2).

Existen muchos fármacos para prevenir o tratar el dolor oncológico, por lo que su uso y vía de administración dependen tanto del tipo de dolor, como de la etiología del mismo.

Un tratamiento adecuado supone comenzar por una evaluación completa del dolor. En el 90 \% de los casos, el dolor puede ser controlado con simples medidas (3).

Para evaluar el dolor normalmente se utilizan escalas de una sola dimensión. La más comúnmente utilizada es la escala visual analógica (EVA), que consiste en una línea recta, de $10 \mathrm{~cm}$ de largo, con sus extremos delimitados por las marcas "ausencia de dolor" y "peor dolor imaginable" representando así la intensidad del dolor como variable continua. Se trata de un método simple, rápido y fiable (4).

La depresión es una comorbilidad frecuente entre los pacientes oncológicos, que tiene un impacto negativo en su calidad de vida. La depresión clínica se define como un síndrome que comprende esencialmente síntomas afectivos (tristeza patológica, abatimiento, irritabilidad, sentimientos de incomodidad e impotencia frente a las demandas de la vida), aunque también puede incluir trastornos cognitivos o incluso síntomas somáticos en mayor o menor grado.

El diagnóstico de la enfermedad generalmente se hace según los criterios del "Manual diagnóstico y estadístico de los trastornos mentales (DSM-IV)", que evalúa la presencia de una serie de síntomas depresivos y su duración (5). Estos síntomas depresivos incluyen estado de ánimo depresivo, trastornos del sueño, alteraciones en el apetito y peso, pérdida de energía, aumento o disminución de la actividad psicomotora, menor capacidad de concentración, sentimientos de culpa y desvalorización y pensamientos suicidas (6).

Si no se trata la depresión conduce al sufrimiento del paciente y a un incremento de la mortalidad y la morbilidad. Esta es la razón principal que justifica el aumento del número de estudios realizados, que buscan establecer criterios diagnósticos fiables y desarrollar instrumentos de evaluación de depresión en pacientes oncológicos.

El objetivo principal de este estudio fue evaluar la relación entre dolor oncológico y depresión, así como determinar qué factores de la historia clínica del paciente podían estar potencialmente asociados al hecho de padecerla.

\section{MÉTODOS}

Se trata de un estudio observacional, retrospectivo, multicéntrico, de casos y controles, llevado a cabo en España en 40 unidades oncológicas, desde octubre de 2007 a marzo de 2008. Se seleccionaron pacientes de edad $\geq 18$ años, se recogió información disponible en su historia clínica de al menos 3 meses de antigüedad, eran pacientes que experimentaron dolor posterior al diagnóstico de cáncer (EVA $\geq 3$ ), y no tenían ningún impedimento físico o psíquico para la cumplimentación del inventario de depresión de Beck (IDB). Todos los pacientes incluidos en el estudio 
otorgaron su consentimiento informado por escrito. Los pacientes fueron excluidos si presentaban alguna de las siguientes situaciones: diagnóstico de depresión anterior al diagnóstico de cáncer, dolor crónico previo al diagnóstico de cáncer, o cualquier enfermedad psiquiátrica previa. El estudio fue aprobado por el comité ético del Hospital Vall D'Hebrón, Barcelona, España.

Los pacientes del estudio fueron clasificados en casos y controles. Se definieron los casos como aquellos pacientes con dolor oncológico y depresión de moderada a grave (IDB ${ }^{3} 8$ ). Los controles, en cambio, fueron aquellos pacientes con dolor oncológico y depresión leve o ausente (IDB £ 7). Cada investigador incluyó aproximadamente 10 pacientes (1:1) que cumplían los criterios de selección anteriormente descritos.

La realización de análisis adicionales sobre qué factores clínicos podían asociarse potencialmente al hecho de padecer depresión, implicó reclasificar la población del estudio en pacientes "deprimidos" y "no-deprimidos", teniendo en cuenta de nuevo la puntuación del IDB. Se construyó un modelo de regresión logística univariante para evaluar la relación entre cada factor de riesgo y la posibilidad de desarrollar una depresión.

Solamente las variables que mostraron significación estadística en el análisis de regresión univariante se utilizaron en el modelo logístico multivariante. Las odds ratio de cada variable fueron redondeadas al número más cercano.

\section{Variables}

Para cada paciente se registraron los siguientes datos retrospectivos (últimos 3 meses anteriores al estudio): características demográficas, historia clínica oncológica, valoración del dolor y tratamientos analgésicos prescritos.

El inventario de depresión de Beck (IDB) $(7,8)$ fue utilizado para evaluar los síntomas de depresión de los pacientes del estudio. El IDB comprende 13 items, puntuados del 0 al 3 desde la peor a la mejor condición de su estado emocional. La puntuación global del IDB se obtuvo sumando las puntuaciones individuales de cada ítem. Así, para puntuaciones de 0 a 4 , se consideró ausencia de depresión o depresión mínima; puntuaciones de 5 a 7 indicaban depresión leve; de 8 a 15, depresión moderada, y 16 o más puntos, depresión grave.

La valoración de la intensidad del dolor se realizó a través de la escala visual analógica (EVA), una escala unidimensional versátil y fácil de utilizar. Se registró la intensidad de dolor experimentado en las últimas 24 horas, en los últimos 7 días y en los últimos 3 meses (desde ausencia de dolor (0) al peor dolor imaginable (10). Para el análisis de los datos obtenidos, se siguieron las siguientes puntuaciones: 0 a 3 implicaba dolor ausente; de 3 a 5, dolor leve; 5 a 7 era indicativo de dolor moderado $\mathrm{y} \geq 7$, dolor intenso.
Se utilizó también una escala verbal categórica (EVC) de intensidad del dolor con 4 categorías: dolor ausente, leve, moderado o intenso.

\section{Análisis estadístico}

Para el análisis de los datos se utilizó el programa estadístico SAS versión 9.1.3.

Para determinar la relación entre la presencia de depresión y diferentes factores de la historia clínica de los pacientes, se utilizó el test de chi-cuadrado y se realizó un análisis de regresión logística multivariante.

Se incluyeron los siguientes variables: sexo, edad, localización del cáncer, estadio y extensión del cáncer, tipo de tratamiento recibido, tiempo de supervivencia, grado de conocimiento de la enfermedad por parte del paciente, características del dolor (tipo de dolor, temporalidad, estacionalidad, origen del dolor) y enfermedades concomitantes.

\section{RESULTADOS}

Se incluyó un total de 276 pacientes en el estudio (138 casos y 134 controles) de los cuales 248 se consideraron pacientes evaluables. Ocho pacientes del grupo caso y 16 del grupo control se excluyeron por no cumplir la totalidad de los criterios de inclusión.

El 52,4 \% de la población estaba constituido por hombres, frente al $47,6 \%$ de mujeres, con una media de edad de 59,0 $(12,4)$ años. Las características demográficas y del dolor de la población total, así como de los grupos caso y control se muestran en las tablas I y II. Los datos de la segunda clasificación de la población, en pacientes deprimidos y no deprimidos, no se muestran debido a su similitud con los resultados de la primera clasificación (casos y controles). Los tumores primarios que tenían una prevalencia de al menos el $10 \%$ se reflejan en la tabla I: tumores pleuropulmonares $(19,8 \%)$, de mama (23\%), digestivos $(25,4 \%)$ y tumores del aparato urinario $(9,7 \%)$. Un mayor porcentaje de pacientes en el grupo control presentaba cáncer de mama (28,8 \%) en comparación con el grupo caso $(17,7 \% ; p=0,0376)$. No se encontraron diferencias estadísticamente significativas entre grupos respecto al estadio y diagnóstico de la enfermedad. Asimismo, los pacientes de ambos grupos recibieron un tratamiento oncológico similar.

La puntuación total media del IDB para el grupo caso fue de 13,1 $\pm 4,9$ puntos y de 3,8 $\pm 2,1$ para el grupo control.

Las características del dolor están resumidas en la tabla II. Las diferencias entre grupos no fueron significativas para ninguna de las variables estudiadas: tipo de dolor ( $\mathrm{p}$ $=0,2406)$, temporalidad $(\mathrm{p}=0,3426)$, estacionalidad $(\mathrm{p}$ $=0,5181)$, origen del dolor $(\mathrm{p}=0,2027)$ y localización. 
TABLA I. CARACTERÍSTICAS DEMOGRÁFICAS Y CLÍNICAS DE LOS PACIENTES

\begin{tabular}{|c|c|c|c|c|}
\hline & \multicolumn{3}{|c|}{ Pacientes (\%) } & \multirow[b]{2}{*}{$p^{l}$} \\
\hline & Total & Casos & Controles & \\
\hline \multicolumn{5}{|l|}{ Datos demográficos } \\
\hline Hombres & $129(52,4)$ & $72(56,3)$ & $57(48,3)$ & 0,2126 \\
\hline Mujeres & $117(47,6)$ & $56(43,8)$ & $61(51,7)$ & \\
\hline Media de edad (DE) & $58,95(12,35)$ & $60,43(11,41)$ & $57,30(13,17)$ & \\
\hline \multicolumn{5}{|c|}{ Localización de los tumores primarios más frecuentes } \\
\hline Pleuropulmonar & $49(19,8)$ & $29(22,3)$ & $20(16,9)$ & 0,2899 \\
\hline Mama & $57(23,0)$ & $23(17,7)$ & $34(28,8)$ & $\mathbf{0 , 0 3 7 6}$ \\
\hline Digestivo & $63(25,4)$ & $36(27,7)$ & $27(22,9)$ & 0,3847 \\
\hline Aparato urinario & $24(9,7)$ & $13(10,0)$ & $11(9,3)$ & 0,8569 \\
\hline \multicolumn{4}{|l|}{ Estadio $T$} & 0,1506 \\
\hline $\mathrm{T} 1$ & $15(7,8)$ & $8(7,9)$ & $7(7,6)$ & \\
\hline $\mathrm{T} 2$ & $45(23,3)$ & $17(16,8)$ & $28(30,4)$ & \\
\hline $\mathrm{T} 3$ & $71(36,8)$ & $42(41,6)$ & $29(31,5)$ & \\
\hline $\mathrm{T} 4$ & $62(32,1)$ & $34(33,7)$ & $28(30,4)$ & \\
\hline \multicolumn{4}{|l|}{ Metástasis } & 0,4466 \\
\hline M0 & $71(30,9)$ & $35(28,7)$ & $36(33,3)$ & \\
\hline M1 & $159(69,1)$ & $87(71,3)$ & $72(66,7)$ & \\
\hline \multicolumn{3}{|l|}{ Tratamiento oncológico } & 0,1233 & \\
\hline Radioterapia & $8(3,5)$ & $6(5,1)$ & $2(1,8)$ & \\
\hline Radioterapia y otros & $1(0,4)$ & & $1(0,9)$ & \\
\hline Radioterapia y quimioterapia & $115(50,7)$ & $61(51,7)$ & $54(49,5)$ & \\
\hline Radioterapia, quimioterapia y otros & $13(5,7)$ & $3(2,5)$ & $10(9,2)$ & \\
\hline Otros & $90(39,6)$ & $48(40,7)$ & $42(38,5)$ & \\
\hline \multicolumn{5}{|l|}{ Tiempo medio de supervivencia (rango) } \\
\hline & $15,0(9-30)$ & $12(6-24)$ & $20(12-36)$ & $\mathbf{0 , 0 0 3 2}$ \\
\hline
\end{tabular}

${ }^{1}$ Chi-squared o Wilcoxon-Mann Whitney, según lo apropiado

El tiempo medio de supervivencia, por el contrario, mostró diferencias significativas entre ambos grupos, siendo mayor en los pacientes del grupo control (20,0 meses) en comparación con los pacientes del grupo caso (12 meses; $\mathrm{p}=0,0032$ ).

La fig. 1 muestra el tratamiento analgésico (opioides y no opioides) recibido por los pacientes durante los últimos 3 meses (Fig. 1A y 1B); el recibido en el momento del estudio (Fig. 1C y 1D), y el tratamiento prescrito durante la visita de estudio (Fig. 1E y 1F). No hubo diferencias estadísticamente significativas entre los grupos, excepto para el uso global de opioides durante los últimos 3 meses (grupo caso $=86,8 \%$ y grupo control $=66,7 \% ; \mathrm{p}=0,0008$ ) y para el uso de fentanilo (grupo caso $=65,2 \%$ y grupo control $=44,2 \% ; p=0,0008)$.

En relación a la intensidad del dolor, según la EVC, un porcentaje similar de pacientes en el grupo caso y en el grupo control experimentaron dolor asociado al cáncer (95,4 vs $94,1 \%$, respectivamente; $\mathrm{p}=0,6422)$.
De acuerdo con la EVA para el dolor, los pacientes en el grupo caso experimentaron una intensidad media del dolor más elevada durante las últimas 24 horas $(5,6[2,9])$ que los pacientes del grupo control $(4,4[2,6] ; p=0,0002)$. Lo mismo ocurrió para la intensidad media de dolor experimentado durante los últimos 7 días (6,1 [2,7] y 5,3 [2,5]; $p$ $=0,0039$, para el grupo caso y control, respectivamente). Por el contrario, no se observaron diferencias significativas entre el grupo caso y el grupo control en cuanto a la intensidad del dolor experimentado durante los últimos 3 meses $(6,6[2,4]$ y $6,4[2,6] ; p=0,5161)$.

Tal y como sucedió con el dolor evaluado según la EVA, el número de horas de dolor difirió entre grupos. El grupo caso experimentó una media de 10,95 $(9,0)$ horas de dolor durante las últimas 24 horas, en comparación con las 8,1 $(8,1)$ horas de media en el grupo control $(p=0,0173)$. De forma similar, la media de horas de dolor durante los últimos 7 días fue superior en los pacientes del grupo caso en comparación con los pacientes del grupo control (5,691 
TABLA II. CARACTERIZACIÓN DEL DOLOR

\begin{tabular}{|c|c|c|c|c|}
\hline & \multicolumn{3}{|c|}{ Pacientes (\%) } & \multirow[b]{2}{*}{$p^{l}$} \\
\hline & Total & Casos & Controles & \\
\hline Tipo de dolor & & & & 0,2406 \\
\hline Superficial & $13(5,2)$ & $4(3,1)$ & $9(7,6)$ & \\
\hline Superficial y visceral u óseo & $2(0,8)$ & & $2(1,7)$ & \\
\hline Superficial y neuropático & $2(0,8)$ & $1(0,8)$ & $1(0,8)$ & 0,29 \\
\hline Visceral u óseo & $195(78,6)$ & $104(80,0)$ & $91(77,1)$ & \\
\hline Visceral u óseo y neuropático & $13(5,2)$ & $6(4,6)$ & $7(5,9)$ & \\
\hline Neuropático & $57(9,3)$ & $15(11,5)$ & $8(6,8)$ & \\
\hline Temporalidad del dolor & & & & 0,3426 \\
\hline Agudo & $33(13,3)$ & $14(10,8)$ & $19(6,1)$ & \\
\hline Crónico & $191(77,0)$ & $105(80,8)$ & $86(72,9)$ & \\
\hline Incidente & $24(9,7)$ & $11(8,5)$ & $13(11,0)$ & \\
\hline Estacionalidad & & & & 0,5181 \\
\hline Permanente & $154(62,3)$ & $84(64,6)$ & $70(59,8)$ & \\
\hline Predominio diurno & $60(24,3)$ & $28(21,5)$ & $32(27,4)$ & \\
\hline Predominio nocturno & $32(13)$ & $18(13,8)$ & $14(12,0)$ & \\
\hline Origen del dolor & & & & 0,2027 \\
\hline Provocado por el tumor & $209(85,7)$ & $113(87,6)$ & $96(83,5)$ & \\
\hline Producido por el tratamiento & $28(11,5)$ & $11(8,5)$ & $17(14,8)$ & \\
\hline No asociado al tumor ni al tratamiento & $7(2,9)$ & $5(3,9)$ & $2(1,7)$ & \\
\hline Localización & & & $1(0,9)$ & \\
\hline Abdominal & $29(11,7)$ & $12(9,2)$ & $17(14,4)$ & \\
\hline Articular & $1(0,4)$ & & & \\
\hline Cabeza & $4(1,6)$ & $4(3,1)$ & & \\
\hline Cadera/coxis/sacro/pelvis & $25(10,1)$ & $12(9,2)$ & $13(11,0)$ & \\
\hline Cervical & $15.0(6,0)$ & $10(7,7)$ & $5(4,2)$ & \\
\hline Dorsal & $27(10,9)$ & $10(7,7)$ & $17(14,4)$ & \\
\hline Espalda & $8(3,2)$ & $4(3,1)$ & $4(3,4)$ & \\
\hline Extremidades inferiores & $22(8,9)$ & $11(8,5)$ & $11(9,3)$ & \\
\hline Extremidades superiores & $12(4,8)$ & $7(5,4)$ & $5(4,2)$ & \\
\hline Faringe/esófago/cavidad oral & $16(6,5)$ & $8(6,2)$ & $8(6,8)$ & \\
\hline Gástrico & $12(4,8)$ & $9(6,9)$ & $3(2,5)$ & \\
\hline Lumbar & $41(16,5)$ & $23(17,7)$ & $18(15,3)$ & \\
\hline Torácico & $23(9,3)$ & $15(11,5)$ & $8(6,8)$ & \\
\hline Óseo & $11(4,4)$ & $6(4,6)$ & $5(4,2)$ & \\
\hline
\end{tabular}

${ }^{1}$ Test de Chi-cuadrado

[2,12] frente a 5,1 [2,3]; $\mathrm{p}=0,0292)$. Cuando se realizó el análisis respecto a los últimos 3 meses, no se encontraron diferencias estadísticamente significativas entre grupos $(67,7[38,6]$ y $61,8[40,1]$, para el grupo caso y control, respectivamente; $\mathrm{p}=0,2165)$. La tabla III muestra la relación entre la puntuación del IDB y de la EVA para el dolor y el número de horas de dolor. Los coeficientes de correlación más altos del IDB se hallaron en la medida de la intensidad del dolor durante las últimas 24 horas $(0,215 ; \mathrm{p}=0,0007)$ y en cuanto a las horas de dolor durante las últimas 24 horas $(0,170 ; p=0,0073)$.
En relación al análisis adicional sobre los factores clínicos que podrían estar potencialmente asociados a padecer depresión, el número total de pacientes después de reclasificar a la población en pacientes "deprimidos" y "no deprimidos" basados en el IDB ( $\geq 5$, y 0-4, respectivamente) para el siguiente análisis fue de 236. Los datos que figuran a continuación, muestran una elevada similitud con respecto a los citados previamente en la primera clasificación, de casos y controles. El 52,1 \% eran hombres y 47,95 \% mujeres, con una media de edad de 59,0 (12,2) años. Los tumores más frecuentes fueron: pleuropulmonar $(20,3 \%)$, de 

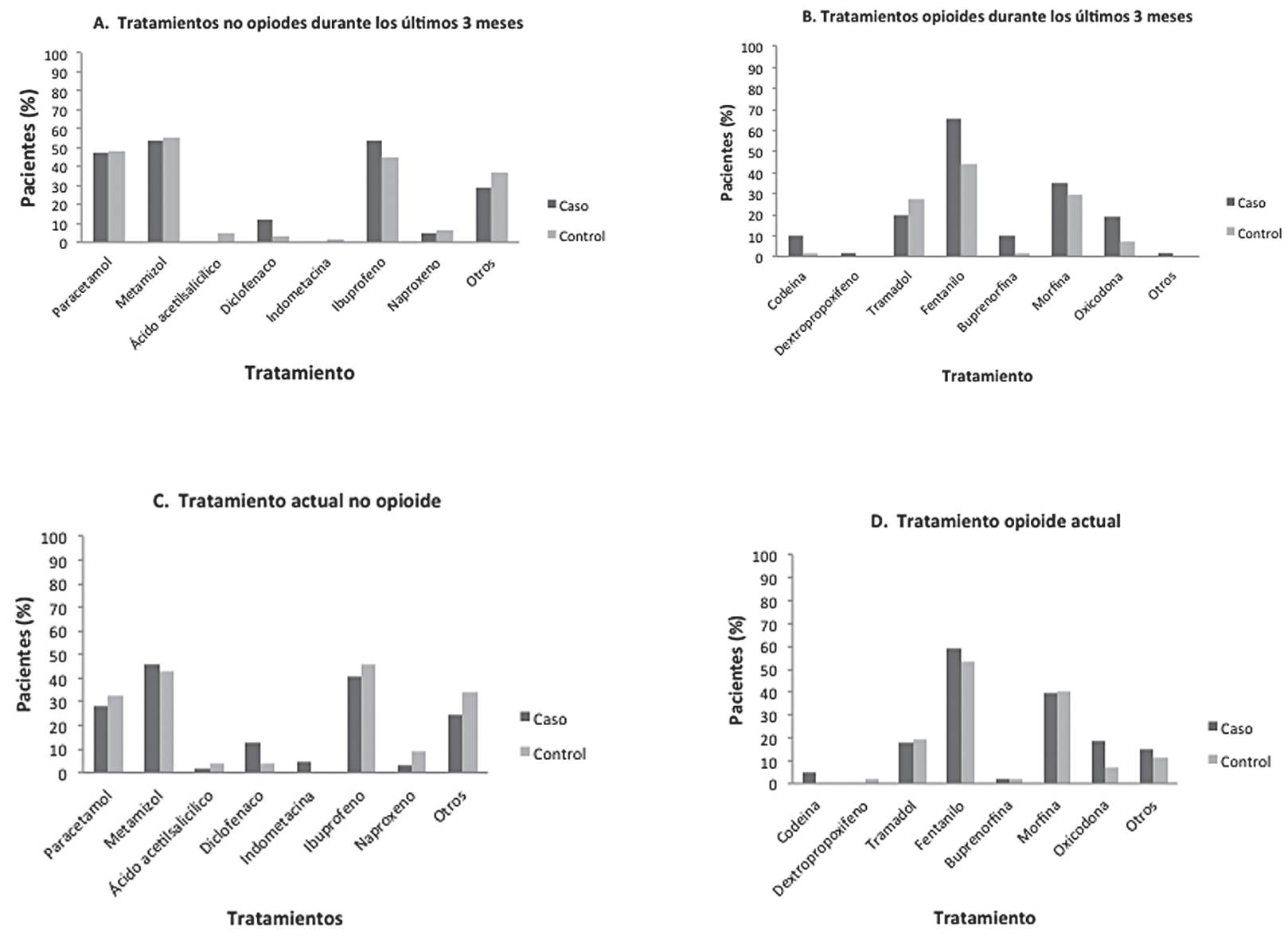

E. Tratamientos no opioides prescritos en la visita de estudio

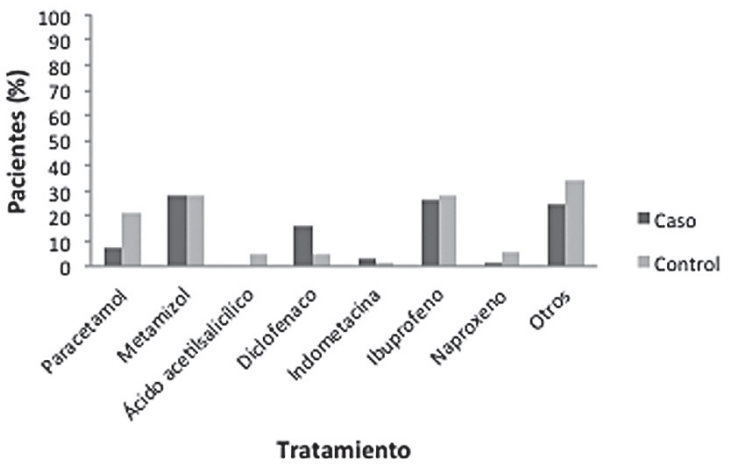

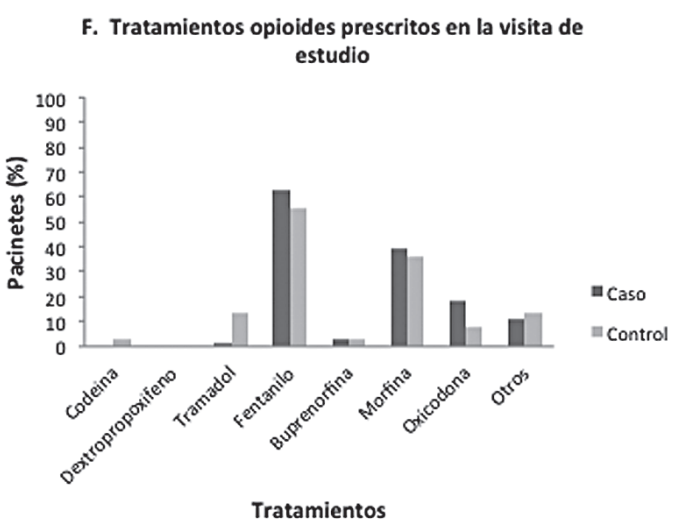

Fig. 1. Tratamientos opioides y no opioides recibidos por los pacientes del grupo control (gris oscuro) y grupo caso (gris claro) durante los últimos 3 meses (A y B), en el momento del estudio (C y D), y prescritos en la visita de estudio (E y F).

mama $(22,8 \%)$, aparato digestivo $(25,7 \%)$ y aparato urinario $(10,1 \%)$. El cáncer de mama fue más frecuente en el grupo de pacientes no deprimidos $(32,8 \%)$ comparado con los pacientes deprimidos $(19,1 \% ; \mathrm{p}=0,0252)$. En cambio, los pacientes con depresión presentaron una incidencia más alta de cáncer digestivo (29,5\%) en comparación con los pacientes no deprimidos $(15,6 \%: \mathrm{p}=0,0303)$. En cuanto al estadio de la enfermedad, los pacientes deprimidos tenían estadios tumorales más avanzados en comparación con los pacientes no deprimidos. Concretamente, en el grupo de los 
pacientes deprimidos hubo un porcentaje más bajo de casos en estadio T2 $(18,2 \%)$ en comparación con el obtenido en el grupo de pacientes no deprimidos (32,0 \%). De la misma manera, el 40,1\% de los pacientes deprimidos tenía cáncer en estadio T4, a diferencia del $14 \%$ encontrado en el grupo de pacientes no deprimidos (valor global $\mathrm{p}=0,0066$ ). No se encontraron diferencias significativas entre grupos en relación al tratamiento oncológico $(\mathrm{p}=0,1833)$. El tiempo medio de supervivencia en los pacientes deprimidos fue inferior (13,0 meses) al observado en los pacientes no deprimidos (20,0 meses, $\mathrm{p}=0,0226)$.

Respecto a la temporalidad del dolor, se observó un porcentaje mayor de dolor agudo en pacientes no deprimidos $(23,4 \%)$ en comparación con los pacientes deprimidos $(8,1$ $\% ; \mathrm{p}=0,0045)$. De forma similar, en cuanto al origen del dolor, se observó mayor dolor asociado al tratamiento en los pacientes no deprimidos $(19,0 \%)$ en comparación con los pacientes deprimidos $(9,4 \% ; \mathrm{p}=0,0487)$. No hubo diferencias estadísticamente significativas entre grupos
TABLA III. COEFICIENTES DE CORRELACIÓN ENTRE LA PUNTUACIÓN DEL INVENTARIO DE DEPRESIÓN DE BECK Y LA EVA PARA EL DOLOR

\begin{tabular}{lcc}
\hline & Beck & $p$ \\
\hline EVA 24 horas & 0,215 & $\mathbf{0 , 0 0 0 7}$ \\
EVA 7 días & 0,134 & $\mathbf{0 , 0 1 4 3}$ \\
EVA 3 meses & 0,043 & 0,5007 \\
$\begin{array}{l}\text { Horas de dolor en las últimas } \\
\text { 24 horas }\end{array}$ & 0,170 & $\mathbf{0 , 0 0 7 3}$ \\
$\begin{array}{l}\text { Horas de dolor en los últimos } \\
\text { días }\end{array}$ & 0,142 & $\mathbf{0 , 0 2 5 4}$ \\
$\begin{array}{l}\text { Horas de dolor en los últimos } \\
\text { 3 meses }\end{array}$ & 0,053 & 0,4056 \\
$\begin{array}{l}\text { Horas de dolor en el último día } \\
\text { con dolor }\end{array}$ & 0,179 & $\mathbf{0 , 0 0 5 2}$ \\
\hline
\end{tabular}

EVA: escala visual analógica.

TABLA IV. VARIABLES PREDICTORAS ASOCIADAS A LA DEPRESIÓN (BDI $\geq 5$ ). MODELO UNIVARIANTE

\begin{tabular}{llccc}
\hline Variable & Nivel & OR & $C I(95 \%)$ & $p$ \\
\hline Sexo & Hombre vs mujer & 1,45 & $(0,82 ; 2,59)$ & 0,2028 \\
Edad & & 1,02 & $(0,99 ; 1,04)$ & 0,1224 \\
Pleuropulmonar & Sí vs No & 1,00 & $(0,49 ; 2,03)$ & 0,9890 \\
Mama & Sí vs No & 0,48 & $(0,25 ; 0,92)$ & $\mathbf{0 , 0 2 6 9}$ \\
Digestivo & Sí vs No & 2,26 & $(1,07 ; 4,78)$ & $\mathbf{0 , 0 3 3 3}$ \\
Aparato urinario & Sí vs No & 1,12 & $(0,42 ; 2,97)$ & 0,8156 \\
Estadio T & T1 vs T4 & 0,35 & $(0,09 ; 1,40)$ & $\mathbf{0 , 0 1 1 3}$ \\
& T2 vs T4 & 0,20 & $(0,07 ; 0,54)$ & \\
Metástasis & T3 vs T4 & 0,25 & $(0,10 ; 0,65)$ & \\
Tratamiento & M0 vs M1 & 0,87 & $(0,46 ; 1,65)$ & 0,6701 \\
Tiempo de supervivencia & & 1,55 & $(0,80 ; 2,98)$ & 0,2953 \\
Tipo de dolor & Otros vs RDT y QMT & 2,99 & $(0,35 ; 25,28)$ & \\
& RDT vs RDT y QMT & 0,99 & $(0,99 ; 1,00)$ & 0,1975 \\
Temporalidad & Neuropático vs visceral & 1,15 & $(0,40 ; 2,29)$ & $\mathbf{0 , 0 2 9 0}$ \\
& Superficial vs visceral & 0,21 & $(0,07 ; 0,68)$ & \\
Estacionalidad & Agudo vs incidente & 0,47 & $(0,15 ; 1,49)$ & $\mathbf{0 , 0 0 5 2}$ \\
& Crónico vs incidente & 1,73 & $(0,65 ; 4,55)$ & \\
Origen & Permanente vs predominio nocturno & 0,92 & $(0,37 ; 2,32)$ & 0,4258 \\
& Predominio diurno vs predominio & 0,61 & $(0,22 ; 1,67)$ & \\
& nocturno & & $(0,00 ; 1,00)$ & 0,1726 \\
& No asociado al tumor ni al tratamiento vs & & $(0,20 ; 1,04)$ & \\
\hline & provocado por el tratamiento & & \\
Producido por el tratamiento vs provocado & 0,46 & & \\
& por el tumor & & &
\end{tabular}


TABLA V. VARIABLES PREDICTORAS ASOCIADAS A LA DEPRESIÓN. MODELO MULTIVARIANTE

\begin{tabular}{llccc}
\hline Variable & Nivel & OR & CI $(95 \%)$ & $p$ \\
\hline Cáncer digestivo & No vs Sí & 0,41 & $(0,17 ; 0,98)$ & 0,0454 \\
Estadio T & T1 vs T4 & 0,40 & $(0,09 ; 1,61)$ & 0,1967 \\
& T2 vs T4 & 0,22 & $(0,08 ; 0,61)$ & $\mathbf{0 , 0 0 3 9}$ \\
& T3 vs T4 & 0,20 & $(0,08 ; 0,53)$ & $\mathbf{0 , 0 0 1 2}$ \\
\hline
\end{tabular}

para las variables tipo de dolor $(\mathrm{p}=0,0876)$ y estacionalidad del dolor $(\mathrm{p}=0,4358)$.

La tabla IV muestra los resultados del modelo de regresión univariante. El hecho de padecer cáncer de mama incrementó el riesgo de sufrir depresión 0,48 veces $(\mathrm{p}=$ $0,0269)$, mientras que tener cáncer digestivo incrementó el riesgo 2,26 veces $(\mathrm{p}=0,0333)$. Un estadio más avanzado de la enfermedad también figuraba entre los factores que incrementaban el riesgo de padecer depresión ( $p=0,0113$ ), siendo los pacientes en estadio $\mathrm{T} 4,0,35$ veces más propensos a padecer depresión en comparación con los pacientes en estadio T1. Para las variables asociadas al dolor en sí mismo, tanto el tipo de dolor como la temporalidad, resultaron factores de riesgo significativos de padecer depresión ( $\mathrm{p}=0,0290$ y $\mathrm{p}=0,0052$, respectivamente).

De todas las variables que resultaron significativas en el modelo de regresión univariante, el análisis multivariante únicamente confirmó el carácter predictivo del cáncer digestivo $(\mathrm{p}=0,0454)$ y del estadio $\mathrm{T}(\mathrm{T} 2$ vs T4; $\mathrm{p}=$ 0,0039 , y T3 vs T4; $p=0,0012$ ) como factores de riesgo de padecer depresión (Tabla V).

\section{DISCUSIÓN}

Este estudio evaluó la relación entre dolor y depresión en pacientes oncológicos con diferentes tipos de cáncer. Los resultados confirman, como en estudios previos, una clara correlación entre ambos factores en pacientes con enfermedades crónicas $(9,10)$ incluyendo las de origen oncológico (11).

Se observa una relación significativa entre dolor y depresión, especialmente cuando el dolor estaba referido a un breve espacio de tiempo. Esta correlación quedó demostrada en el estudio, en base a la puntuación de la EVA (durante las últimas 24 horas y durante los últimos 7 días) con respecto a la puntuación del IDB.

La conexión entre dolor y depresión parece residir en el mecanismo fisiológico subyacente en ambos procesos. El desequilibrio en la concentración de los neurotransmisores serotonina y noradrenalina, causante de depresión, es también responsable de una disminución del umbral de la sensibilidad al dolor. Los pacientes deprimidos, por lo tanto, tienen una mayor sensibilidad nociceptiva (12). A pesar de que la prevalencia de depresión varía considerablemente, de 0 a $58 \%$, el cáncer se asocia a un alto nivel de depresión (13). Los estudios realizados por Lansky y cols. (14), Zimmerman y cols. (15) y Kelsen y cols. (16) evaluaron la correlación entre dolor oncológico y depresión y observaron una relación entre la intensidad del dolor y el estado psicológico del paciente. Sin embargo, y como una limitación de este estudio, se requieren análisis adicionales para evaluar no solamente esta asociación, sino también para comprobar cómo un tratamiento adecuado del dolor oncológico puede repercutir en el estado psicológico del paciente. En este sentido, existe un elevado interés por estudiar el impacto del dolor en la calidad de vida de los pacientes oncológicos (17).

Los pacientes que padecen depresión, de moderada a grave, tienden a experimentar una disminución de su calidad de vida, pues pueden verse altamente afectadas sus actividades sociales y laborales cuando coexisten dolor crónico y depresión (18).

Bajo dolor intenso, los pacientes oncológicos tienen el deseo de adelantar su muerte (11). Además, los autores del mismo estudio observaron que las mejorías en la capacidad funcional asociada al dolor oncológico no variaban dicho deseo, pero sí lo hacían las estrategias centradas en mejorar el estado anímico del paciente. Las implicaciones de estos hallazgos son críticas, sobre todo si se tiene en cuenta que la depresión se encuentra infratratada con frecuencia en los pacientes oncológicos.

Los resultados del estudio D-PRESS muestran la necesidad de identificar a los pacientes en riesgo de padecer depresión, para así proporcionar una aproximación individual que permita abordar y tratar tanto el estado físico, como las necesidades emocionales del paciente oncológico.

\section{AGRADECIMIENTOS}

Los autores agradecen a todos los investigadores su participación en el estudio, así como a Eva Mateu y Anna Ramírez de RPS España su colaboración en la edición del manuscrito.

Este trabajo fue financiado por Janssen-Cilag, promotor del estudio. 


\section{CORRESPONDENCIA:}

Joan Carulla

Hospital Vall D’Hebron

Paseeig de la Vall D’Hebron, 119-129

08035, Barcelona.

Tlfn: 607434759

jcarullatorrent@gmail.com

\section{BIBLIOGRAFÍA}

1. Berry PH, Chapman CR, Covington EC, et al. Pain: current understanding of assessment, management, and treatments. National Pharmaceutical Council. 2004. Disponible en www.ampainsoc.org. Último acceso 4 de abril de 2005.

2. Torres LM. El tratamiento del dolor como un derecho de todos. Rev Soc Esp Dolor. 2005;12:399-400.

3. Sanz Ortiz J. Evaluación clínica del dolor en pacientes con cáncer. En: Díaz-Rubio E (editor). Tratamiento del dolor en oncología. Madrid. Editorial You\&Us. 2003. p. 59-69.

4. Olsen S, Nolan MF, Kori S. Pain measurement. An overview of two commonly used methods. Anesthesiol Rev. 1992;19:11-5.

5. Keller MB, Hanks DL, Klein DN. Summary of the DSM-IV mood disorders field trial and issue overview. Psychiatr Clin North Am. 1996;19:1-28.

6. Williams JW, Hitchcock Noël P, Cordes JA, et al. Is this patient clinically depressed? JAMA. 2002;287:1160-70.

7. Beck AT, Ward CH, Mendelson M, et al. An inventory for measuring depression. Arch Gen Psychiatr. 1961;4:561-71.

8. Beck AT, Rush AJ, Shaw BF, et al. Cognitive Therapy of Depression. New York: Guilford Press. 1979.
9. Chou K. Reciprocal relationship between pain and depression in older adults: Evidence from the English longitudinal study of ageing. Journal of Affective Disorders. 2007; 102(1):115-23.

10. Jann M, Slade J. Antidepressant Agents for the Treatment of Chronic Pain and Depression. Pharmacotherapy. 2007;27 (11):1571-87.

11. O'Mahony S, Goulet J, Kornblith A, et al. Desire for Hastened Death, Cancer Pain and Depression: Report of a Longitudinal Observational Study. J Pain Symptom Manage. 2005;29(5):446-57.

12. Bailey KP. Physical symptoms comorbid with depression and the new antidepressant duloxetine. J Psychosoc Nurs Ment Health Serv. 2003;41:13-8.

13. Massie MJ. Prevalence of depression in patients with cancer. J Natl Cancer Inst Monogr. 2004;(32):57-71.

14. Lansky SB, List MA, Herrmann CA, et al. Absence of major depressive disorder in female cancer patients. J Clin Oncol. 1985;3:1553-60.

15. Zimmerman L, Story KT, Gaston-Johansson F, et al. Psychological variables and cancer pain. Cancer Nurs. 1996; 19:44-53.

16. Kelsen DP, Portenoy RK, Thaler HT, et al. Pain and depression in patients with newly diagnosed pancreas cancer. J Clin Oncol. 1995;13:748-55.

17. So WK, Marsh G, Ling WM, et al. The symptom cluster of fatigue, pain, anxiety, and depression and the effect on the quality of life of women receiving treatment for breast cancer: A multicenter study. Oncol Nurs Forum. 2009; 36(4):E205-14.

18. Bair MJ, Robinson RL, Katon W, et al. Depression and pain comorbidity: a literature review. Arch Intern Med. 2003; 163(20):2433-45. 